\title{
SOBRE LA ESTRUCTURA SINTÁCTICO-DISCURSIVA DE LAS CONSTRUCCIONES DE REFORMULACIÓN
}

\author{
Agustín Vera LuJÁN \\ Universidad Nacional de Educación a Distancia (UNED)
}

\section{Introducción}

Durante los últimos decenios, los estudios del discurso vienen interesán -dose regularmente por la investigación de las relaciones que se producen entre las diferentes unidades discursivas; unas relaciones que han recibido distintas denominaciones según los modelos discursivos de que se trate; entre otras, relaciones retóricas ${ }^{1}$, relaciones de coherencia ${ }^{2}$, predicados retóricos ${ }^{3}$, o proposiciones

${ }^{1}$ Véanse, a este respecto, W. C. Mann y S. A. Thompson, «Rhetorical Structure Theory: a framework for the analysis ot texts», IPRA. Papers in Pragmatics, 1, 1987, págs. 79-105; W. C. Mann y S. A. Thompson, «Rhetorical Structure Theory: Towards a funcional theory of text organization», Text, 8, 3, 1988, págs. 243-281; W. C. Mann, Ch. M. Matthiessen y S. A. Thompson, «Rhetorical Structure Theory and text analysis», en W. C. Mann y S. A. Thompson (eds.), Discourse Description: Diverse Linguistic Analyses of a Fund-Raising Text, John Benjamins, Amsterdam y Filadelfia, 1992, págs. 39-78; y N. Asher y A. Lascarides, Logics of Conversation, Cambridge University Press, 2003.

$2 \mathrm{~J}$. R. Hobbs, On the coherence and structure of discourse, Technical Report, csli -85-37, Center for the Study of Language and Information, Stanford University, Stanford, 1985.

${ }^{3}$ J. E. Grimes, The thread of discourse, Mouton, La Haya, 1975; W. C. Mann y S. A. Thompson, «Rhetorical Structure Theory: a framework for the analysis ot texts», o «Rhetorical Structure Theory: Towards a funcional theory of text organization»; C. Smith, Modes of Discourse: The Local Structure of Texts, Cambridge University Press, 2003.

AnMal, XXXIX, 2016-2017, págs. 179-202 
relacionales $^{4}$, y han sido postuladas en número muy diverso según los diferentes modelos 5 .

A partir especialmente de los estudios sobre el discurso oral, se viene prestando gran atención a un conjunto de relaciones discursivas que se engloba dentro de lo que, a partir de Gülich y Kotschi' se denomina reformulación; una operación por la cual el emisor de un discurso, tras haber dado una determinada forma lingüística a un concepto o a una serie de ellos, procede a una nueva formulación posterior de los mismos: los reformula, produciéndose de esta forma una relación discursiva entre los elementos inicialmente formulados y los reformulados a continuación que ha sido objeto de numerosos estudios, a partir de las investigaciones pioneras de G. Antos ${ }^{7}$ sobre el concepto de formulación, y con posterioridad, sobre todo, de los trabajos Gülich y Kotschi ${ }^{8}$, o los de Roulet ${ }^{9}$.

Los estudios sobre la reformulación han subrayado su carácter de operación discursiva no relacionable directamente con factores gramaticales o léxicos ${ }^{10}$, su naturaleza básicamente enunciativa ${ }^{11} \mathrm{y}$, más específicamente, su condición metalingüística' ${ }^{12}$, o metadiscursiva ${ }^{13}$, pues las relaciones que se establecen en este caso no tienen que ver con las que afectan a los eventos denotados, sino a las que establecen entre sí los elementos del discurso en el proceso para su misma construcción como tal.

Los estudios sobre la reformulación se han interesado muy especialmente por el análisis de los denominados marcadores y operadores de reformulación ${ }^{14}$;

${ }^{4}$ W. C. Mann y S. A. Thompson, «Assertions from discourse structure», Proceedings of 11th Annual Meeting of the Berkeley Linguistics Society, Berkeley, 1985.

${ }_{5}^{5}$ Para un amplio resumen de distintas propuestas al respecto, véase J. Garrido, «Relaciones de discurso», Pandora, revue d' etudes hispaniques, 7, 2007, págs. 305-332.

${ }^{6}$ E. Gülich y T. H. Kotschi, «Les marqueurs de reformulation paraphrastique», Cahiers de Linguistique Française, 5, 1983, págs. 305-351. De los mismos autores véanse también, P. Bange (ed.), «Les actes de reformulation dans la consultation 'La dame de Caluire'», L'analyse des interactions verbales. La Dame de Caluire: une consultation, Lang, Berna, 1987, págs. 15-81, y «Discourse Production in Oral Communication. A study Based on French», en U. Quasthoff (ed.), Aspects of Oral Communication, De Gruyter, Berlín, 1995, págs. 30-66.

${ }^{7}$ G. Antos, Grundlagen einer Theorie des Formulierens, Niemeyer, Tubinga, 1982.

${ }^{8}$ E. Gülich y T. H, Kotschi, «Les marqueurs de reformulation paraphrastique».

${ }^{9}$ E. Roulet, «Completude interactive et mouvements discursifs», Cahiers de Linguistique Française, 7, 1986, págs. 189-206, y, del mismo autor, «Completude interactive et connecteurs reformulatifs», Cahiers de Linguistique Française, 8, 1987, págs. 111-140.

${ }^{10}$ Insiste en la necesidad de separar estos ámbitos C. Fuchs, «La paraphrase entre la langue et le discourse», Langue Française, 53, 1982, págs. 22-33.

${ }^{11}$ C. Fuentes, «Conclusivos y reformulativos», Verba, 20, 1993, págs. 171-198.

${ }_{12}$ M. Murat y B. Cartier Bresson, «C'est á dire ou la reprise interprétative», Langue Française, 73, 1987, págs. 5-15.

${ }^{13}$ E. Roulet, «A modular approach to discourse structures», Pragmatics, 7, 2, 1997, pags. 125-146; A. Briz, «Los conectores pragmáticos en español coloquial (II): su papel metadiscursivo», Español Actual, 59, 1993, págs. 39-56, y, del mismo autor, «Coherencia y cohesión en la conversación coloquial», Gramma-temas, Contextos, 2, 1997, págs. 9-43.

14 Para la definición lexicográfica de estos elementos, véanse L. Santos, Diccionario de partículas, Luso-Española de Ediciones, Salamanca, 2003; C. Fuentes, Diccionario de 
es decir, por los elementos que señalan en el discurso la presencia de dicha relación entre dos elementos, que pueden, respectivamente, encontrarse explícitos, o implícito el primero de ellos ${ }^{15}$. Gracias a estas investigaciones disponemos de detalladas clasificaciones de tales elementos basadas en los rasgos semánticos que los caracterizan.

No ha faltado tampoco en estas investigaciones la preocupación por la caracterización de las estructuras sobre las que descansa la reformulación, destacando a este respecto propuestas de naturaleza semántico argumentativa muy influyentes como las de E. Roulet ${ }^{16}$, continuadas por distintos autores ${ }^{17}$, que fundamentan la distinción entre reformulación paráfrastica $(\mathrm{RP})$ y no parafrástica (RNP) no solo en la equivalencia semántico-informativa o en la ausencia de ella que se produce, respectivamente, en cada una de estos tipos de reformulación, sino también en la correspondencia de cada una de ellas con una estructura discursiva diferente: coordinada en el primero de los casos, y subordinada en el segundo; con la particularidad de que, en este, la función de elemento principal le correspondería al elemento reformulador (RDOR) y la de subordinado al reformulado (RDO) ${ }^{18}$.

El objetivo de nuestro trabajo es la caracterización sintáctica de la estructura discursiva de la reformulación; el estudio de tales construcciones desde una perspectiva sintáctica que no ha sido dominante a la hora de describir su estructura, especialmente en el dominio de los estudios sobre la comunicación oral, y que creemos contribuirá a enriquecer la imagen de su funcionamiento con los resultados de una perspectiva explicativa diferente de la semántico-argumentativa, más habitual. A estos efectos, tras pasar revista en epígrafe 2 a lo esencial de los planteamientos metodológicos a este respecto de algunas de las posiciones más influyentes, especialmente, dentro de los estudios sobre

conectores y operadores del español, Arco/Libros, Madrid, 2009, y A. Briz, S. Pons y J. Portolés (eds.), Diccionario de partículas discursivas del español, <http://www.dpde.es/>.

${ }^{15}$ E. Roulet, «Completude interactive et connecteurs reformulatifs», señala esta doble posibilidad, y M. A. Martín Zorraquino y J. Portolés, «Los marcadores del discurso», en I. Bosque y V. Demonte (eds.), Gramática descriptiva de la lengua española, 3, 1999, Espasa, Madrid, págs. 4051-4215, utilizan esta doble denominación.

${ }^{16}$ E. Roulet, loc. cit.

17 Véase especialmente C. Rossari, «Projet pour une typologie des opérations de reformulation», Cahiers de Linguistique Française, 11, 1990, págs. 345-359. La influencia metodológica de Roulet alcanza, en diferentes formas, a otros investigadores como, entre otros, M. Casado «Los operadores discursivos es decir, esto es, o sea y a saber en español», Lingüistica Española Actual, 13, 1991, págs. 87-116; J. Portolés, «Sobre la organización interna de las intervenciones», en A. Briz et alii, Pragmática y gramática del español hablado, Universidad de Valencia, Departamento de Filología Española, Valencia, 1996, págs. 203-214; P. Garcés, «Los marcadores de recapitulación y reconsideración en el discurso», Revista de Investigación Lingüística, 6, 1, 2003, págs. 111-141, y de la misma autora, «Marcadores de corrección y rectificación en los textos escritos», Revista de Investigación Lingüística, 13, 2010, págs. 87-105.

${ }^{18}$ En sentido diferente, Luis Cortés postula que el papel de segmento discursivo subordinado correspondería al elemento reformulador. Véase su artículo «Las unidades del discurso oral», Boletín de Lingüística, 17, 2002, págs. 7-29. 
el discurso oral, contrastaremos la imagen que de ellos resulta sobre el funcionamiento de la reformulación con la que se puede obtener desde una perspectiva sintáctico-funcional. Para ello, en el epígrafe 3 , tras la explicitación de algunos conceptos metateóricos básicos (relativos tanto al tipo de unidades discursivas que es posible/necesario diferenciar para un estudio en profundidad del funcionamiento de la reformulación, como a la naturaleza y planos de consideración de las relaciones sintácticas que operan en el funcionamiento de las construcciones discursivas), mostraremos cómo la reformulación puede ser descrita, en términos sintácticos, como una operación discursiva que se concreta en estructuras sintácticas de tipo paratáctico, o coordinado, e identificaremos los distintos niveles y unidades discursivas en que se da tal operación. Postularemos, en esencia, que la reformulación puede ser entendida como un tipo de construcción sintáctica cuyos constituyentes presentan, como es característico en las estructuras coordinadas, autonomía o independencia mutuas. Finalmente, en el apartado de conclusiones, el epígrafe 4, destacaremos algunas de las que se desprenden de la perspectiva de análisis elegida, haciendo especial hincapié en la nueva imagen general de la reformulación que es posible trazar cuando es analizada desde una perspectiva sintáctico-discursiva como la que planteamos. Fundamentalmente, la consideración de los distintos tipos de reformulación habitualmente reconocidos como pertenecientes a un único tipo sintáctico abstracto de construcción discursiva coordinada, y la idoneidad del mismo para la función comunicativa esencial de la reformulación.

\section{Aproximaciones semántico-argumentativas a la estructura de las cons- trucciones de reformulación}

La reformulación es un mecanismo discursivo que ha recibido una especial atención en los modelos, inicialmente al menos, centrados en el análisis del discurso oral, pero el interés por este mecanismo, o por algunos de los tipos de relaciones discursivas a que puede dar lugar no ha faltado tampoco en modelos diferentes, de distinta naturaleza, que han desarrollado también diversas propuestas sobre la estructura de las secuencias de reformulación. Como intentaremos mostrar, en ambos casos la descripción de la estructura de las construcciones de reformulación parece haber obedecido fundamentalmente a criterios semánticos que no logran poner de manifiesto de manera adecuada las características fundamentales de este tipo de mecanismo.

En el dominio de los modelos del discurso oral, uno de los más influyentes es el de E. Roulet ${ }^{19}$, cuya caracterización de la estructura de la reformulación descansa fundamentalmente sobre la distinción entre RP y RNP20, entendidas,

${ }^{19}$ E. Roulet, «Completude interactive et connecteurs reformulatifs», pág. 115.

${ }^{20}$ En realidad, Roulet reserva el nombre de reformulación exclusivamente para la reformulación no parafrástica, la que define por coincidir con un movimiento de reformulación retroactivo. E. Roulet, loc. cit., pág. 111. 
respectivamente, como una relación que se da entre dos elementos o secuencias de elementos que se postulan como equivalentes, y aquella otra que afecta a elementos entre los que existe una cierta distancia conceptual. Es la diferencia que se plantearía entre las expresiones de (1) y (2) ${ }^{21}$ :

(1) Es un tipo raro, o sea, no me gusta.

(2) No me han escuchado y me han ignorado; en resumen, me han tratado fatal.

(1) y (2) mostrarían, en efecto dos tipos de estructuras diferentes. En la primera, el constituyente inmediato reformulado, «Es un tipo raro», y el reformulador, «no me gusta», no mantienen ninguna relación jerárquica, dada su equivalencia semántico-conceptual. Por ello, son considerados estructuralmente equivalentes y vinculados por una relación mutua de coordinación. Por el contrario, en (2), RDO, «No me han escuchado y me han ignorado»y RDOR, «en resumen, me han tratado fatal», como sucede en toda RNP, mantienen entre sí una distancia conceptual que Roulet hace sinónimo de relación jerárquica, por lo que considera a dichos constituyentes como afectados por una relación de subordinación, con la particularidad de que el papel de constituyente principal corresponde al RDOR, pues es considerado como el segmento de mayor relevancia informativa, y el de subordinado al RDO. De manera característica, la relación que afecta a los constituyentes de (2) es concebida por Roulet 22 como de «subordinación retroactiva» pues, en el proceso de elaboración de la secuencia, el elemento inicial, al ser combinado con el posterior, perdería su condición de enunciado autónomo y, retroactivamente, se convertiría en subordinado.

Una característica de las operaciones de reformulación señalada por Roulet que ha mantenido su vigencia en planteamientos descriptivos posteriores, reflejada en distinciones conceptuales como la de marcador/operador ${ }^{23}$, es la de que el mecanismo en cuestión puede tomar la forma, en el caso de las RNP, de una relación cuyo primer elemento, RDO, se encuentre implícito. Ello implicaría que uno de los modos de manifestación de las operaciones de reformulación

${ }^{21}$ En general, los autores que distinguen entre RP y RNP dividen estas dos clases, especialmente a la segunda, en ulteriores subtipos no siempre coincidentes. Véase, para un útil resumen de diferentes posiciones al respecto y S. Pons, «Un solo tipo de reformulación», Cuadernos AISPI, 2, 2003, págs. 151-170, para el que se basa fundamentalmente en S. Murillo Ornat, A Contribution to the Pragmalinguistic Contrastive Study of Explicatory Reformulative Discourse Markers in Contemporary Journalistic Written English and Spanish, Tesis Doctoral, Universidad de Zaragoza, 2007.

22 E. Roulet, «Completude interactive et connecteurs reformulatifs», pág. 115.

${ }^{23}$ M. A. Martín Zorraquino y J. Portolés («Los marcadores del discurso», pág. 4072) distinguen este doble funcionamiento en los elementos que señalan la reformulación, basándose en la distinción realizada por Ducrot entre conectores argumentativos y operadores argumentativos. Véase O. Ducrot, «Operateurs argumentatifs et visée argumentative», Cahiers de Linguistique Française, 5, 1983, págs. 7-36. La posibilidad de que el primer elemento de la reformulación se encuentre implícito es sostenida también por E. Roulet, loc. cit., pág. 119. 
sería (3), junto a los ya señalados de (1) y (2). Se postulan de esta manera como posibles dos modos de representación superficial de un solo esquema estructural de reformulación (4): los representados en (4a) y (4b):

(3) encontraremos un nuevo espacio en torno a los libros donde compartir con los niños y jóvenes nuestros respectivos hallazgos y preferencias literarios. Porque se trata, en definitiva, de que lean para que sigan leyendo y, así, vayan trazando su propio itinerario lector.

(CORPES XXI, Equipo Peonza, El rumor de la lectura, Anaya, Madrid, 2001).

(4) [RDO-RDOR]

(4a) / -RDOR/

(4b) /RDO-RDOR/

Los planteamientos metodológicos de Roulet han tenido una gran fortuna posterior en numerosos modelos de análisis del discurso, y han influido, en lo relativo a la conceptuación general de la organización interna de las estructuras de reformulación, en las propuestas de tipologías de relaciones de RNP de otros muchos investigadores ${ }^{24}$. Tales planteamientos vienen a coincidir también, en lo esencial, con los de otros modelos lingüístico-discursivos más específicamente centrados en el análisis del discurso escrito. Así, por ejemplo, con los de Longacre, quien, a la hora de clasificar las unidades que denomina parágrafos - que, en términos generales, se pueden considerar equivalentes a las intervenciones de Roulet ${ }^{25}$-, reconoce la existencia de una subclase de tales unidades a la que denomina parágrafos de paráfrasis ${ }^{26}$. Estos se definen como compuestos por varias oraciones que, variando en su forma, remiten en todo caso a un contenido semejante. Desde el punto de vista estructural, se comportarían en dos modos diferentes: unos, como (5), a los que denomina paráfrasis de tesis equivalentes, como estructuras coordinadas, pues sus constituyentes mantendrían entre sí relaciones de equivalencia, y los restantes - paráfrasis antonímica (6); paráfrasis de amplificación (7), y paráfrasis de resumen o conclusión (8) - estarían estructurados en virtud de relaciones jerárquicas de

${ }^{24}$ C. Rossari, «Projet pour une typologie des opérations de reformulation», págs. 345-352; de la misma autora, Les opérations de reformulation, Peter Lang, Berna, 1994; véanse también A. Briz, «Las unidades de la conversación», RILCE, 16/2, 2000, págs. 225-246; A. Briz y A. Hidalgo, «Conectores pragmáticos y estructura de la conversación», en M. A. Martín Zorraquino y E. Montolío Durán (eds.), Los marcadores del discurso. Teoría y análisis, Arco/ Libros, Madrid, 1998, págs. 121-142; S. Pons, op. cit.; P. Garcés, «Los marcadores de recapitulación y reconsideración en el discurso»; de la misma autora, «Marcadores de corrección y rectificación en los textos escritos»; Grupo Val.Es.Co, «Las unidades del discurso oral. La propuesta Val.Es.Co de segmentación del discurso de la conversación coloquial», Estudios de Lingüística del Español, 35, 1, 2014, págs. 11-71, entre otros.

${ }_{25}$ Para esta propuesta en relación con el párrafo como unidad discursiva, véase A. Vera, «El párrafo como unidad discursiva», Estudios de Lingüística, 26, 2012, págs. 343-358.

${ }^{26}$ R. E. Longacre, The Grammar of Discourse, Plenum Press, Nueva York, 1996, pág. 13. 
subordinación, por lo que es posible identificar en ellos unos elementos como dominantes y otros como auxiliares ${ }^{27}$.

(5) I've never met such a prejudiced man anywere. I've never known such a complete bigot in all my experience.

(6) He's one of the most reliable men we've ever had on the job. He's not always trying to evade responsability like so many of our employees.

(7) They took care of his needs. They sobered him up, fed him, clothed him, and gave him a place to stay.

(8) They sobered him up, fed him, clothed him, and gave him a place to stay. They took care of his needs.

Planteamientos similares podemos encontrarlos dentro de la denominada Teoría de las estructuras retóricas (RST, en sus siglas inglesas), desde la que se postula también la existencia de dos esquemas estructurales de reformulación (restatement). Uno, caracterizado porque la relación entre sus elementos es la de núcleo-satélite, basada en la subordinación, y donde el elemento en función nuclear describe una determinada situación que es reformulada por el satélite; otro, denominado reformulación multinuclear, cuyos elementos o constituyentes, ambos de función nuclear, se considera que son de importancia similar con respecto a los fines del hablante ${ }^{28}$.

Por lo que se refiere a las estructuras de las construcciones correspondientes, las posiciones teóricas mencionadas a este respecto guardan, por tanto, las semejanzas reflejadas en el esquema (9):

(9)

\begin{tabular}{|l|l|l|}
\hline RELACIÓN ESTRUCTURAL & \multicolumn{1}{|c|}{ CoORDINACIÓN } & \multicolumn{1}{c|}{ SUBORDINACIÓN } \\
\hline Roulet & -Reformulación parafrástica & -Reformulación no parafrástica \\
\hline Longracre & -paráfrasis de tesis equivalente & $\begin{array}{l}\text {-Paráfrasis antonímica } \\
\text {-Paráfrasis de amplificación } \\
\text {-Paráfrasis de resumen o conclu- } \\
\text { sión }\end{array}$ \\
\hline RST & -Reformulación multinuclear & -Reformulación \\
\hline
\end{tabular}

${ }^{27}$ R. E. Longacre, «Two hypotheses regarding text generation and analysis», Discourse Processes, 12, 1989, pág. 415.

$28<$ http://www.sfu.ca/rst/08spanish/definiciones.html.>. 
Entre las investigaciones orientadas fundamentalmente al estudio del discurso oral podemos encontrar, no obstante, conceptuaciones diferentes sobre la estructura de la reformulación como una operación discursiva que descansa sobre un único tipo de estructura. Es el caso de la propuesta de L. Cortés, para quien todos los subtipos de reformulaciones obedecerían a un mismo esquema estructural según el cual los elementos RDO y RDOR estarían vinculados por una relación de subordinación, que se establece entre un constituyente director, que cumple una función nuclear en la construcción, y un constituyente subordinado, que cumple la función de margen o submargen. Un ejemplo del mismo autor ${ }^{29}$ que refleja el tratamiento comentado para el caso de una RP (señalada por $o$ sea), además de incluir otras relaciones diferentes de la de reformulación, es (10):

Bueno, yo en mi casa estoy a gusto // pero me falta una habitación más para los niños / que están los tres en un cuarto // o sea, poner los dos más pequeños en uno y al mayor en otro // entonces: si me toca la lotería lo emplearía en eso. ///

(10i)

ESQUEMA SINTÁCTICO: núcleo + margen + submargen + conclusión

FUNCIÓN SEMÁNTICA: afirm. + restr. + explicat. + conclusión

CONECTORES: pero o sea entonces

El análisis de la reformulación por parte de Cortés coincide en lo esencial con el de otros modelos discursivos que, de manera general, reconocen la influencia de Grimes ${ }^{30}$ al considerar este tipo de relación discursiva como un mecanismo de subordinación. Así, autores como Versley y Gastel ${ }^{31}$ distinguen entre las relaciones discursivas la que denominan expansión, que incluye a la de interpretación (con la que se relacionaría el resumen, en esta perspectiva), y la elaboración, con la que sería preciso conectar la reformulación. En todos estos casos de expansión, se trataría de relaciones entre dos elementos, en los que el segundo aporta detalles sobre el primero, pero sin que, a su vez, su presencia sea indispensable para el sentido de este.

Estos planteamientos coinciden esencialmente, en lo que ahora nos interesa, con los encontrables en otros modelos, como la Teoría de la representación del discurso estructurado ${ }^{32}$ (SDRT en sus siglas inglesas), en la cual, aunque no se opera tampoco con un inventario de relaciones donde sea posible identificar un equivalente para todas las que se distinguen habitualmente en modelos como el de Roulet, sí se encuentran dos tipos de relaciones que se incluyen

${ }^{29}$ L. Cortés, op. cit., pag. 18.

${ }^{30}$ J. E. Grimes, The Thread of Discourse, Mouton, La Haya, 1975.

31 Y. Versley y A. Gastel, «Linguistic Tests for Discourse Relations in the TüBa-D/Z Corpus of Written German», Dialogue and Discourse, 4, 2, 2013, págs. 142-173.

${ }^{32}$ N. Asher y A. Lascarides, Logics of Conversation, Cambridge University Press, 2013. 
habitualmente en la reformulación en los modelos del discurso oral de inspiración ginebrina, resumen y reformulación, las cuales son consideradas como ejemplos de la relación denominada elaboración, y conceptualizados como relaciones de subordinación, una relación, como señala Asher, «where the second constituent $[. .$.$] describes in more detail some aspect of some eventuality or$ some fact described in the first constituent $\rangle^{33}$.

A pesar de la utilización de conceptos como los de núcleo, margen, coordinación o subordinación, los planteamientos de base sobre los que descansan las categorizaciones de la estructura de las construcciones de reformulación a las que hemos pasado rápida revista no son de naturaleza sintáctica, sino informativa o semántica, y no dejan de plantear, por otra parte, algunos problemas, tanto desde un punto de vista descriptivo como explicativo.

El modelo desarrollado por Roulet y otros investigadores influidos por sus planteamientos utiliza, en efecto, los conceptos de núcleo/satélite como nociones de carácter semántico-informativo, haciendo depender la condición de núcleo de un constituyente de su relevancia informativo-argumentativa, y la de satélites de su carácter secundario o no relevante en el mismo plano semántico-informativo. La determinación de cuándo un constituyente discursivo desempeña la función de núcleo se decidiría, en esta línea, comprobando su condición de elemento esencial para la continuación del discurso en que la construcción de que se trate está inserta, como recuerda S. Pons ${ }^{34}$. Se trata de un criterio que suscita problemas para su aplicación pues, si bien parece funcionar en casos como los de los ejemplos aducidos por Pons ${ }^{35}$, no es menos cierto que no es de utilidad en otros casos, donde la continuación de un discurso puede, como en (11), anclarse más bien sobre los contenidos o informaciones que aportan en una construcción los constituyentes subordinados o satélites.

A. ¿Por qué no viniste a la comida?

B. No fui porque no me gusta Pedro. Siempre me ha tratado mal, va hablando mal de mí a todo el mundo y me perjudica siempre que puede.

Tampoco está exento de problemas como mecanismo empírico para la determinación del núcleo de una construcción el sugerido por Roulet ${ }^{36}$ : la posibilidad de eliminación de los contenidos discursivos en función no nuclear sin alterar con ello el sentido final del discurso de que se trate. Como ha puesto

${ }^{33}$ N. Asher, "Context in Content Composition», en R. Kempson, T. Fernando y N. Asher (eds.), Handbook of Philosophy of Science, 14: Philosophy of Linguistics, North Holland, Ámsterdam, 2012, pag. 264.

${ }^{34}$ S. Pons, op. cit., pág. 159.

35 S. Pons, loc. cit., págs. 160-161.

${ }^{36}$ E. Roulet, «Échanges, interventions et actes de langage dans la structure de la conversation», Études de Linguistique appliquée, 44, 1981, págs. 7-39. 
de manifiesto Van Dijk ${ }^{37}$, el sentido global de un discurso puede ser, en ocasiones, el resultado de la selección, en una secuencia de unidades coordinadas que contienen información de la que denomina constitutiva, y no accidental, de una de las unidades, que posee mayor relevancia informativa que las anteriores, pero esto no le resta a las demás su carácter de elementos nucleares de la construcción desde un punto de vista estructural. Así pues, el criterio de eliminación no ayuda a identificar en forma no ambigua los constituyentes no nucleares de una construcción, pues en el proceso de identificación del sentido básico de un texto o discurso es posible que elementos nucleares del mismo puedan verse afectados por operaciones macroestructurales de eliminación al igual que los satélites.

Planteamientos en esencia no diferentes son los que podemos encontrar, a este respecto, en el marco de la RST, donde el carácter nuclear de un determinado constituyente en una construcción se pone en relación con la importancia o centralidad que le es asignada por el autor/emisor ${ }^{38}$, aspecto empíricamente no fácil tampoco de determinar. Decidir, en el plano concreto del análisis, si el papel de un constituyente es nuclear o no sería, pues, una operación sujeta a los problemas descriptivos mencionados en el párrafo anterior.

Caracterizaciones de la estructura de las construcciones de reformulación como las que comentamos presentan, a nuestro modo de ver, otro problema de adecuación, de naturaleza explicativa: no son capaces de formalizar un nivel estructural en el que las construcciones de RP y RNP puedan ser representadas en términos de un conjunto de propiedades estructurales comunes.

Por lo que a los planteamientos de propuestas como las mencionadas de Cortés o la SRDT se refiere, sus criterios básicos son también de naturaleza semántico-conceptual. En esencia, salvadas las diferencias de detalle, en ambas se postula que las operaciones de reformulación, tanto las de RP como las de RNP vienen a ser un mecanismo de modificación de una primera formulación, por lo que son conceptualmente secundarias o dependientes respecto de aquella, que las precede lógicamente ${ }^{39}$. Un punto de vista semejante se ajusta difícilmente a la conceptuación de la reformulación como una operación originada en el deseo del hablante de lograr una nueva y preferida expresión lingüística de determinados conceptos para los que construye, de este modo, dos formulaciones equivalentes, y parece de difícil aplicación especialmente a modalidades de la reformulación como la corrección o rectificación, en la medida en

37 T. A. Van Dijk, Texto y contexto, Cátedra, Madrid, 1980, págs. 215-216.

38 Véase en $<$ http://www.sfu.ca/rst/08spanish/definiciones.html. $>$ el esquema relativo a las relaciones multinucleares. En él, dentro de las condiciones que deben respetar los núcleos, se establece que «un elemento es una repetición de otro al que se encuentra unido; los elementos son de importancia similar con respecto a los fines de A», y en lo relativo a la intención de A, «L reconoce la repetición de los elementos unidos». A y $L$ representan al autor y al lector del texto, cuyas intenciones son fundamentales para la definición de las unidades.

${ }^{39}$ N. Asher y L. Vieu, «Subordinating and coodinating discourse relations», Lingua, 115, 2005, pág. 598. 
que en esta el segundo miembro de la construcción, RDOR, se postula como el segmento relevante, cuyo valor informativo anula denotativamente al del constituyente RDO.

\section{La estructura sintáctica de las construcciones de reformulación}

A pesar, como hemos visto, de la existencia de caracterizaciones de la estructura de las construcciones de reformulación basadas en la utilización de conceptos como los de núcleo y margen o coordinación y subordinación, aquellas a las que hemos pasado revista en el epígrafe 2 se basan en criterios de naturaleza semántico-informativa, y no en un punto de vista estrictamente sintáctico, que ayudaría, en nuestra opinión, a superar algunas de las inadecuaciones de tales caracterizaciones y, de otro lado, enriquecería nuestra comprensión del funcionamiento de estas construcciones. Nuestro objetivo central en este trabajo consistirá en esbozar las líneas básicas de una aproximación semejante a las construcciones de reformulación.

Naturalmente, el análisis de las construcciones de reformulación, y, en general, del discurso, desde una perspectiva sintáctica no supone postular la existencia, en dicho dominio lingüístico, de una sintaxis gramaticalizada, lo que no quiere decir que no sea posible plantear la existencia en su caso de lo que Stati denominara una sintaxis transfrástica ${ }^{40}$, cuya operatividad puede ser fundamentada en los mismos principios generales que nos son familiares tras las investigaciones realizadas sobre el componente sintáctico-gramatical; unos principios a los que podemos suponerles, por consiguiente, un carácter semiótico o cognitivo básico.

A imagen y semejanza del proceder en la sintaxis oracional, la sintaxis discursiva puede ser concebida como la disciplina que se ocupa del estudio de las relaciones que se producen entre los constituyentes inmediatos de las unidades del discurso ${ }^{41}$. Respecto de estas, entre los distintos modelos discursivos existentes, en este trabajo nos basaremos en el modelo de organización discursiva desarrollado por el Grupo Val.Es.Co ${ }^{42}$ en relación con el discurso monológico, $\mathrm{y}$, dados nuestros intereses actuales, nos centraremos en las unidades inferiores a la intervención, por tratarse de aquellas en las que puede observarse de manera prototípica la operatividad de los mecanismos de reformulación.

${ }^{40}$ S. Stati, Le transphrastique, Puf, París, 1990, pág. 136.

${ }^{41}$ Para una síntesis de los aspectos fundamentales sobre los que descansa una sintaxis oracional funcional, extrapolables, en nuestra opinión, en lo esencial, para la descripción del funcionamiento sintáctico de las unidades del discurso, véase G. Rojo y T. Jiménez Juliá, Fundamentos del análisis sintáctico funcional, Lalia, Universidad de Santiago de Compostela, 1998.

${ }^{42}$ Grupo Val.Es.Co, op. cit. Dentro del ámbito hispánico, han formulado también propuestas diferentes sobre la forma de un modelo discursivo, y sus unidades distintos autores. Entre ellos, véanse C. Fuentes, Lingüistica pragmática y análisis del discurso, Arco/Libros, Madrid, 2000; L. Cortés, op. cit:; y J. Portolés, Pragmática para hispanistas, Síntesis, Madrid, 2004. 
Dentro del modelo citado, en el que las distintas unidades se conciben como jerarquizadas y sometidas a la operatividad del mecanismo de la recursividad, la intervención, asociada prototípicamente a los cambios de locutor, se caracteriza como la unidad estructural de mayor rango jerárquico ${ }^{43}$. Su constituyente inmediato es el acto, la «mínima unidad de acción e intención» ${ }^{44}$, y en la estructura de este se reconoce, a su vez, como constituyente inmediato al subacto, caracterizado por «constituir un segmento informativo identificable» ${ }^{45}$. Desde nuestro punto de vista, resultaría conveniente contar, en el plano de las unidades informativas, no solo con el subacto, sino con otro tipo de unidades menores, constituyentes inmediatos de este, a los que podríamos denominar unidades discursivas elementales, que coincidirían con los conceptos elementales que integrarían informativamente un subacto, pues las operaciones de reformulación se dan en ocasiones entre unidades discursivas menores que el subacto, entre unidades que funcionarían como constituyentes inmediatos de este. En lo sucesivo, nos referiremos a dichas unidades utilizando las siguientes representaciones:

(12)

Intervención: I

Acto: A

Subacto: SA

Unidad discursiva elemental: UDE

De acuerdo con este catálogo de unidades discursivas monológicas, la operatividad de los mecanismos de reformulación se manifestaría en ejemplos como los de (13a-c), donde no se recoge ningún caso afectado por el mecanismo de la recursividad, y que se concretan, respectivamente, en las unidades reflejadas en (13a'-c'):

(13a) Eso es bullying, es decir, acoso.

(13b) Tiene vocación de médico, o sea, quiere curar a la gente.

(13c) Te pido que me traigas esa silla; mejor dicho, te lo ordeno.

(13a') $\mathrm{I}>\mathrm{A}>\mathrm{SA}>\mathrm{UDE}-\mathrm{UDE}$

(13b') $\mathrm{I}>\mathrm{A}>\mathrm{SA}-\mathrm{SA}$

(13c') $\mathrm{I}>\mathrm{A}-\mathrm{A}$

Caracterizar sintácticamente las relaciones de reformulación en tanto que relaciones sintácticas discursivas hace necesario también concretar la naturaleza de estas relaciones entre los constituyentes inmediatos de las unidades discursivas señaladas. Estas relaciones pueden concebirse como equivalentes de las

\footnotetext{
${ }^{43}$ Grupo Val.Es.Co, loc. cit., pág. 15.

${ }^{44}$ Loc. cit., pág. 36.

${ }^{45}$ Loc. cit., pag. 53.
} 
funciones hjelmslevianas de constelación, determinación e interdependencia ${ }^{46}$, en la línea de la caracterización de los mecanismos sintácticos de coordinación y subordinación oracionales propuesta originariamente por García Berrio ${ }^{47}$ y desarrollada especialmente, en distinta forma, por Rojo ${ }^{48}$, A. Narbona ${ }^{49}$ y Jiménez $J_{u l i a ́}^{50}$, en el ámbito hispánico ${ }^{51}$. De esta forma resulta posible operar con unos conceptos funcionales vaciados de contenidos semántico-conceptuales, confiriendo a los conceptos tradicionales de núcleo y margen valores estrictamente sintácticos, como los que rigen la definición de las funciones hjelmslevianas ${ }^{52}$ :

a. Constelación: relación entre dos variables. Los dos elementos se comportarán funcionalmente como núcleo de la construcción de que son constituyentes.

b. Determinación: relación entre una constante y una variable. De los dos elementos el primero se comportará funcionalmente como núcleo y el segundo como margen.

c. Interdependencia: relación entre dos constantes. Los dos elementos se comportarán funcionalmente como núcleo de la construcción de que son constituyentes.

Una caracterización plenamente adecuada de las relaciones sintácticas discursivas, hace necesario también tomar en consideración la distinción entre dos perspectivas en el tratamiento o descripción de unidades y relaciones: las que denominaremos, siguiendo a S. Gutiérrez ${ }^{53}$ (1983), abstracta y concreta, y en virtud de las cuales podríamos hablar de unidades y relaciones sintácticas abstractas y de unidades y relaciones sintácticas concretas.

${ }^{46}$ L. Hjelmslev, Prolegómenos a una teoría del lenguaje, Gredos, Madrid, 1972 (edic. original 1943).

${ }^{47}$ A. García Berrio, Bosquejo para una descripción de la frase compuesta en español, Universidad de Murcia, 1970.

${ }^{48}$ G. Rojo, Cláusulas y oraciones, Verba, Anuario Gallego de Filología, Anejo 14, Universidad de Santiago de Compostela, 1978.

49 A. Narbona, Las subordinadas adverbiales impropias en español (bases para su estudio), Ágora, Málaga, 1989; del mismo autor, Subordinadas adverbiales impropias en español, II: Causales y finales, comparativas y consecutivas, condicionales y concesivas, Ágora, Málaga, 1990.

${ }^{50}$ T. Jiménez Juliá, La coordinación en español. Aspectos teóricos y descriptivos, Verba, Anuario Gallego de Filología, Anejo 39, Universidad de Santiago de Compostela, 1995

${ }^{51}$ Aunque con orientaciones particulares, el concepto de interdependencia ha sido utilizado en otros modelos lingüísticos de manera central. Véanse, entre otros, a este respecto R. E. Longacre, «Sentence Structure as a Statement Calculus», Language, 46, 4, 1970, págs. 783-815; W. A. Foley y R. D. Van Valin, Funcional Syntax and Universal Grammar, Cambridge University Press, 1984; o P Hopper y E. C. Traugott, Grammaticalization, Cambridge University Press, 2004.

52 Como es habitual, representaremos gráficamente estas relaciones en el modo siguiente: con $«+»$, las relaciones de constelación, con « $\rightarrow »$, las de determinación, y con $« \leftrightarrow »$ las de interdependencia.

${ }^{53} \mathrm{~S}$. Gutiérrez, «La determinación inmanente de las funciones en sintaxis», Contextos, 2, 1983, págs. 41-58. 
La distinción mencionada, que Gutiérrez remite a la equivalente en la tagmémica norteamericana entre slot y tagmeme $e^{54}$, sugiere la necesidad de diferenciar, a la hora de caracterizar unidades y relaciones sintácticas, entre su análisis en tanto que unidad/relación que pertenece a un determinado tipo o clase general distinto de otros también generales (la unidad oración, por ejemplo, en tanto que unidad de dicho nivel, frente a otras unidades abstractas, de niveles lingüísticos diferentes, como el sintagma, o las relaciones sintácticas oracionales en el marco de dicha unidad abstracta) y aquel otro que toma en consideración sus características como unidad/relación concreta dentro de un tipo general o abstracto, con unas características que la hacen distinta de otras pertenecientes al mismo tipo general (una oración personal, en la que, por tanto, ha de estar presente la relación que denominamos habitualmente 'sujeto', frente a una impersonal, carente de dicha relación, por ejemplo).

Una distinción semejante es de gran utilidad en el dominio de la sintaxis oracional, al permitir conciliar sin contradicción apreciaciones tan aparentemente incompatibles como, por ejemplo, que la oración es una unidad cuyo elemento nuclear es el tradicionalmente denominado predicado, y que oraciones concretas como (14) o (15) son otros tantos ejemplos de oraciones bien formadas, siendo así que solo el primero de los ejemplos (14), parecería estar en consonancia con el postulado de que el predicado es el núcleo de la oración (de hecho, es el único constituyente de la oración de nuestro ejemplo), mientras que parecería en contradicción con (15), enunciado oracional donde se comprueba la presencia obligatoria de un constituyente en función de sujeto que no puede ser eliminado sin que el enunciado resultante esté mal formado. Esta aparente contradicción se revela, en efecto, como inexistente, situada en el marco metodológico mencionado, puesto que la definición de la oración como construcción sintáctica integrada por un único elemento nuclear (y otros, en consecuencia, marginales) se postularía de la oración unidad abstracta; es decir, se trataría de una condición que ha de cumplir cualquier enunciado para tener la condición de oración-abstracta, para tener la condición de oración, mientras que las oraciones concretas (14) y (15) presentarían unas relaciones sintácticas que serían debidas a las capacidades/exigencias sintagmáticas de sus constituyentes: el verbo llover no admite, en empleos prototípicos, sujeto, por lo que su uso en una oración conlleva que esta se comporte estructuralmente como una oración-concreta impersonal, mientras que hablar, al contrario, no puede emplearse sin sujeto, por lo que su uso supone necesariamente la construcción de una oración-concreta personal:

(14) Llueve.

(15) Pedro habló.

La distinción entre unidades discursivas abstractas/concretas, en combinación con la conceptuación de las relaciones sintácticas como relaciones

\footnotetext{
${ }^{54}$ En concreto, S. Gutiérrez remite a J. T. Platt, Grammatical relations and grammatical meaning, North-Holland, Ámsterdam, 1971, págs. 1-5.
} 
funcionales en sentido hjelmsleviano, permite describir en forma adecuada el funcionamiento sintáctico de las unidades del discurso. En el plano abstracto, estas obedecen a dos esquemas de construcción diferentes, dependiendo de que las relaciones que sus constituyentes virtuales establezcan entre sí sean de determinación o de constelación. La determinación es la relación que vincula a los constituyentes inmediatos de enunciados como (16) a (18); la constelación a los de los ejemplos de (19) a (21).

La existencia de intervenciones como (16a) o (16b) pone, en efecto, de manifiesto que la estructura sintáctica de una intervención abstracta responde a un esquema estructural en el que los posibles constituyentes inmediatos estarían sujetos a una relación de determinación, ya que es posible la existencia de una intervención abstracta integrada por un único constituyente A (16a), al cual le correspondería por tanto la función de núcleo de la unidad. A su vez, la existencia de actos como (17a) o (17b) nos indica que las construcciones de este nivel discursivo responden al mismo esquema de relaciones de determinación, pues son posibles actos abstractos como (17b), con un único constituyente SA, por tanto, nuclear. Finalmente, se puede comprobar a través de enunciados como (18b) que un SA abstracto descansa también estructuralmente sobre relaciones funcionales de determinación, pues una unidad abstracta de dicho nivel puede ser también una construcción con un único constituyente UDE. Los enunciados de nuestros ejemplos obedecen, por tanto, a los esquemas funcionales representados en (16c)-(18c).

(16a) Ven. Necesito tu ayuda.

(16b) Ven.

(16c)

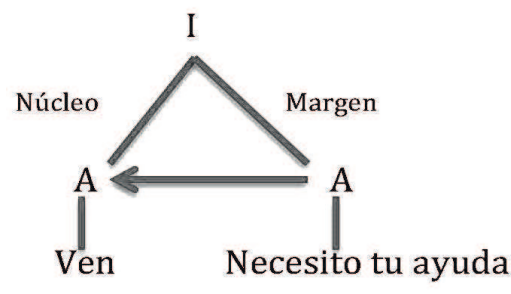

(17a) Me voy porque tengo prisa.

(17b) Me voy.

(17c)

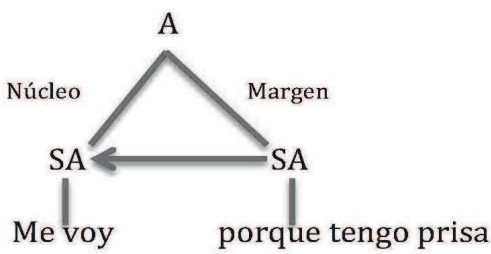


(18a) Ha venido tu prima María.

(18b) Ha venido tu prima.

(18c)

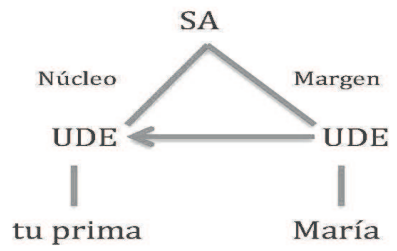

La otra configuración posible de la estructura sintáctica de las unidades discursivas abstractas a que nos referíamos es la que tiene lugar cuando sus constituyentes inmediatos establecen entre sí una relación de constelación; una relación en la que aquellos se comportan funcionalmente como variables, de manera que ninguno de ellos presupone al otro o depende de él, funcionando, pues, ambos como núcleos de la construcción correspondiente. Esta estructura es la que subyace a (19), (20) o (21), donde se muestran tres construcciones abstractas pertenecientes a otros tantos niveles discursivos formadas por dos constituyentes inmediatos cualquiera de los cuales podría ser el único integrante de la construcción. En el caso de (19) se trataría de un A compuesto, en el de (20) de un SA compuesto, y en el de (21) de una UDE compuesta. Representamos en (19c)-(21c) la estructura relacional de dichas unidades discursivas.

(19) ¡Sube al piso de arriba y bájame un libro!

(19a) ¡Sube al piso

(19b) ¡Bájame el libro!

(19c)

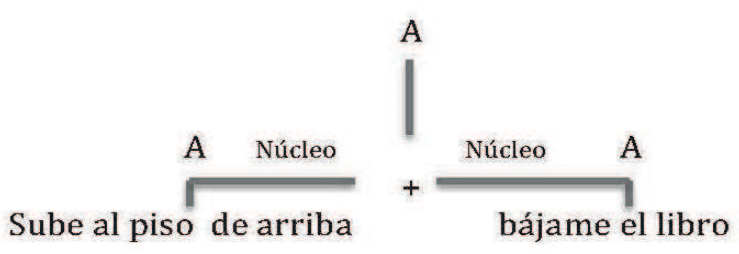

(20) Se ha levantado tarde y no ha llegado a tiempo a la cita.

(20a) Se ha levantado tarde.

(20b) No ha llegado a tiempo a la cita.

(20c)

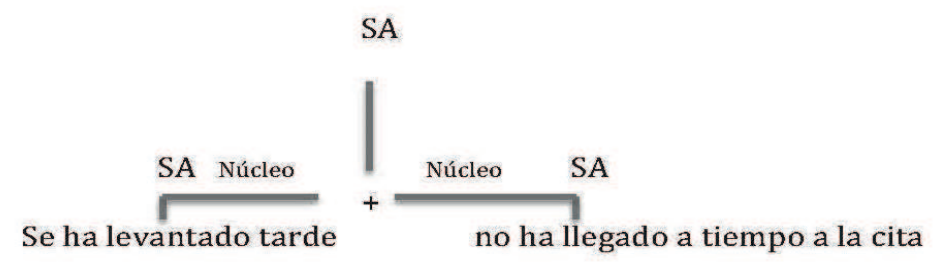


(21) Ha venido tu prima, María.

(21a) Ha venido tu prima.

(21b) Ha venido María

(21c)

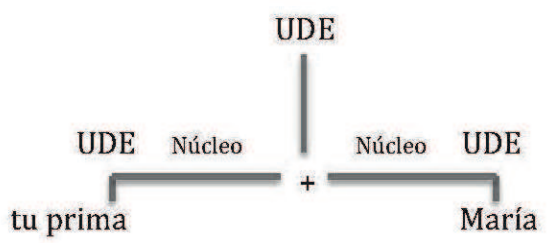

Por lo que al plano de las unidades y relaciones concretas se refiere, se trata en este caso de atender a las características que singularizan, dentro de una unidad/ relación abstracta, a sus distintos tipos específicos (oración personal, impersonal, transitiva, intransitiva..., dentro de la oración-abstracta; sujeto agente, paciente, experimentador..., dentro de la relación sujeto-abstracto, etc.). En el plano específico de las unidades discursivas, los tipos de unidades encontrables resultan de la operatividad de dos esquemas sintácticos posibles: unos basados en relaciones de determinación, como sucedería en (22), y otros en relaciones de interdependencia, como en (23). En el primero de ellos encontramos, en efecto, dos A como constituyentes inmediatos de una intervención, de los que el primero funciona como una especie de marco del segundo; es decir, posee un carácter meramente circunstancial ${ }^{55}$. Establece para con el segundo constituyente una relación de determinación. Por el contrario, enunciados como (23) son ejemplos de un caso en el que la relación entre ambos constituyentes da origen a un sentido de 'causalidad' en virtud del cual percibimos al primer constituyente como conectado con un papel o función de 'causa' y al segundo como su 'efecto'. Dado que esta relación causal requiere inexcusablemente de ambos elementos, causa y efecto, es necesario concluir que la relación que vincula a los dos constituyentes inmediatos del enunciado es una relación de interdependencia.

(22) El día era soleado. Estuvimos en la playa toda la mañana.

(23) Me ha tratado mal siempre. Ahora no voy a ir a su boda.

Centrándonos sin más en la caracterización sintáctica de la reformulación como operación discursiva, según lo indicado se habrán de tomar en consideración las relaciones funcionales operativas en las construcciones que resultan de tal operación. El objeto de una sintaxis de la reformulación consistirá prioritariamente, pues, en el estudio de tales relaciones, y no en el de los marcadores que suelen venir asociados a las construcciones donde aquellas se ponen

${ }_{55}$ W. C. Mann y S. A. Thompson, «Rhetorical Structure Theory: Towards a funcional theory of text organization», pág. 250, denominan a esta relación circunstancia. 
de manifiesto, y cuya presencia, por otra parte, no es imprescindible ${ }^{56}$, en las situaciones y los contextos apropiados, salvo en las reformulaciones de distanciamiento. Así, en efecto, recurriendo a algunos de los tipos de reformulación propuestos por Martín Zorraquino y Portolés ${ }^{57}$, mientras que podemos encontrar reformulaciones explicativas como (22) donde la presencia de un marcador es opcional — véanse (24a) vs. (24b) — y recapitulativas o de rectificación — como (25) y (26) - donde ambas alternativas son igualmente posibles, sin que la ausencia de un marcador de reformulación impida necesariamente la presencia de dicho contenido discursivo en las expresiones relacionadas sintácticamente en tal forma, la ausencia de un marcador de distanciamiento parece indispensable para que el enunciado correspondiente pueda transmitir específicamente dicho contenido reformulativo, como comprobamos en lo inadecuado a este respecto de la alternancia (27a)-(27b).

(24a) Pedro quiere ser médico, dedicarse a curar a la gente.

(24b) Pedro quiere ser médico, es decir, dedicarse a curar a la gente.

(25a) Tomamos cerveza, vino, champagne, coñac; todo el alcohol imaginable.

(25b) Tomamos cerveza, vino, champagne, coñac; en resumen, todo el alcohol imaginable.

(26a) Deme una barra de pan..., dos, dos barras.

(26b) Deme una barra de pan..., o mejor, dos, dos barras.

(27a) *No pienso darte 100 euros. Te daré 10.

(27b) No pienso darte 100 euros. En todo caso te daré 10.

La consideración de la reformulación en tanto que estructura sintáctico-discursiva como la señalada obliga a poner en cuestión la idea de considerar adecuada, desde un punto de vista sintáctico, la existencia de reformulaciones carentes del primer miembro de la estructura, como las de (3), postulando, como sostiene Roulet ${ }^{58}$, que dicho miembro es recuperable por encontrarse albergado en la memoria discursiva de los comunicantes. Parece imposible asumir esta posibilidad en una descripción de la estructura abstracta de las construcciones que estudiamos, pues ello obligaría a aceptar la existencia de dos esquemas sintácticos alternativos de reformulación. Creemos preferible la alternativa de considerar que la presencia de uno de los denominados operadores de reformulación en tales casos no debe interpretarse como señal de la existencia de un mecanismo sintáctico de reformulación, sino como la presencia de un enunciado estructuralmente simple cuyo sentido reformulador se localiza en el plano pragmático-inferencial, como consecuencia de los valores anafóricos de dicho operador, sin que con ello sea necesario suponer la elisión de un primer

56 Véase, por ejemplo, C. Rossari, «Projet pour une typologie des opérations de reformulation», pág. 348.

${ }^{57}$ M. A. Martín Zorraquino y J. Portolés, op. cit., pág. 4082.

58 E. Roulet, «Une description modulaire de l'organisation topicale d'un fragment d'entretien», Cahiers de Linguistique Française, 18, 1996, pág. 19. 
miembro no expreso, cuyos contenidos concretos no alcanzamos a ver cómo se lograría recuperar en gran número de ocasiones.

Desde un punto de vista sintáctico parece adecuado mantener la consideración de la reformulación como una operación discursiva que descansa sobre la estructura sintáctica no simple de las unidades en las que opera. En tanto que unidades abstractas, las relaciones que vinculan a sus constituyentes inmediatos son relaciones de constelación; relaciones entre dos constituyentes inmediatos de una construcción que se comportan como variables; es decir, que ni se presuponen ni mantienen entre sí relaciones de dependencia unilateral ${ }^{59}$. Recurriendo a la clasificación de marcadores de reformulación de M. A. Martín Zorraquino y J. Portolés ${ }^{60}$, podemos comprobar semejante comportamiento funcional en los diversos tipos de reformulación reconocidos por dichos autores, que, a los efectos actuales, podemos considerar equivalentes de otras tantas construcciones sintácticas reformulativas.

Las construcciones abstractas que, siguiendo la denominación de los marcadores correspondientes por parte de Martín Zorraquino y Portolés, denominaremos explicativas, se presentan en unidades del tipo A, SA y UDE, respectivamente (28a), (29a) y (30a) ${ }^{61}$, con las características propias de los constructos estructurados en virtud de relaciones de constelación: el papel desempeñado por la construcción puede ser desempeñado por cada uno de sus constituyentes, como pone de manifiesto la buena formación de (28b)-(30b):

(28a) Murió en Bruselas en 1973. Es decir: murió en el año en que los militares chilenos dieron el golpe de Estado.

(CORPES XXI, Roberto Bolaño, «Vagabundo en Francia y Bélgica», en Putas asesinas, Anagrama, Barcelona, 2001)

(28b) Murió en el año en que los militares chilenos dieron el golpe de Estado.

(29a) La situación, a primera vista, parecía embarazosa, pero para mí fue como un segundo o un tercer nacimiento, es decir, para mí fue el inicio de la esperanza y al mismo tiempo la conciencia desesperada de la esperanza.

(CORPES XXI, Roberto Bolaño, «El retorno», en Putas asesinas, Anagrama, Barcelona, 2001)

(29b) La situación, a primera vista, parecía embarazosa, pero para mí fue el inicio de la esperanza y al mismo tiempo la conciencia desesperada de la esperanza.

59 Una conceptuación semejante las estructuras que tienen su manifestación gramatical en la aposición es la de C. Fuentes, que las define como construcciones constelativas sin nexo. Véanse C. Fuentes, «De nuevo sobre la aposición», Verba, 16, 1989, págs. 209-236 y «Conclusivos y reformulativos», Verba, 20, 1993, pág. 174.

${ }^{60}$ M. A. Martín Zorraquino y J. Portolés, op. cit., págs. 4081-4082.

${ }^{61}$ Los ejemplos han sido extraídos de RAE, Corpus del español del siglo XXI (CORPES XXI), $<$ http://web.frl.es/corpes/view/inicioExterno.view>.[consultada el 6-11-2016]. 
(30a) Pero el nuevo monarca tenía otros asuntos que atender previamente. Estaba consciente de que el ritual de toma de posesión sólo servía para hacerlo gobernante, o sea, convertirse de soldado en rey.

(CORPES XXI, Ernesto Bondy Reyes, «Ocaso del imperio emplumado», en Viaje de Retorno, hasta Sabina y otros relatos, Iberoamericana, Tegucigalpa, 2001)

(30b) Pero el nuevo monarca tenía otros asuntos que atender previamente. Estaba consciente de que el ritual de toma de posesión sólo servía para convertirse de soldado en rey.

El mismo tipo de construcciones abstractas coordinadas, es decir, basadas en relaciones de constelación entre sus constituyentes inmediatos, se da en los casos de reformulación de distanciamiento. En (31a)-(33a) se ofrecen ejemplos de tales construcciones de los niveles A, SA y UDE, respectivamente, y en (31b)-(33b) el resultado de la transformación de tales enunciados en otros simples, que probarían la existencia de una relación de constelación entre los constituyentes inmediatos de los primeros:

(31a) Un libro puede informar, convencer, emocionar, consolar, liberar, deleitar, desasosegar, desesperar... En definitiva, se puede salir de un libro completamente transformado.

(CORPES XXI, Equipo Peonza, El rumor de la lectura, Anaya, Madrid, 2001)

(31b) En definitiva, se puede salir de un libro completamente transformado.

(32a) saben que muchas veces el hábito lector es anterior a la automatización, que es con la práctica lectora continua como va disminuyendo su esfuerzo y van adquiriendo soltura y agilidad; saben, en definitiva, que es con la lectura habitual como se van consiguiendo esos automatismos. (CORPES XXI, Equipo Peonza, El rumor de la lectura, Anaya, Madrid, 2001)

(32b) Saben, en definitiva, que es con la lectura habitual como se van consiguiendo esos automatismos.

(33a) [...] lo tienes todo: una consulta dental con rayas irregulares color café excremento, identidad robada, una vida que no es tuya, una amante muda que no es «una» sino «un», en definitiva una vida agitada [...].

(CORPES XXI, Juan Carlos Quiroz, El anticristo y los malditos del lunes 13, Secretaría de Comunicación y Cultura, Santiago de Chile, 2001)

(33b) $[\ldots]$ lo tienes todo: en definitiva una vida agitada $[\ldots]$.

Las relaciones abstractas de reformulación que se dan en el caso de las construcciones de rectificación son también del tipo constelativo, y dan, por tanto, lugar a construcciones coordinadas en los niveles de A, SA y UDE, como 
mostrarían los ejemplos (34a)-(36a), que admiten equivalentes como (34b)-(36b), donde uno solo de tales constituyentes desempeña el papel de la construcción coordinativa como conjunto:

(34a) Quizá pronto, efectivamente, sepamos qué aspectos concretos de su naturaleza dependen de lo innato y cuáles de lo social. O mejor dicho, quizá podamos desentrañar la compleja maraña de mutuas influencias ambientales y sociales que nos forman.

(CORPES XXI, Pedro Jorge Romero, «Gemelos: entorno, genes y el misterio de la identidad de Lawrence Wright», pjorge.com. pjorge. com: pjorge.com, 22-09-2001)

(34b) Quizá podamos desentrañar la compleja maraña de mutuas influencias ambientales y sociales que nos forman.

(35a) Yo le pedí a Quiteño una explicación sobre la creencia de que Salarrué se hacía pichiche. ...1, me lo explicó a su manera o, mejor dicho, me relató otro cuento: [...].

(CORPES XXI, Eugenio Martínez Orantes, El vuelo del togoroz: lo jamás escrito sobre Salarrué, Editorial Martínez Orantes, El Salvador, 2001)

(35b) Yo le pedí a Quiteño una explicación sobre la creencia de que Salarrué se hacía pichiche. ...1, me relató otro cuento: [...]-

(36a) [...] llevaba unos tenis gastados de tanto andar, unos tenis que en el círculo de mis amistades o mejor dicho en el círculo de los hijos de algunas de mis amistades, estarían desde hace mucho tiempo sepultados en el closet o abandonados en un basurero.

(CORPES XXI, Roberto Bolaño, «Dentista», en Putas asesinas, Anagrama, Barcelona, 2001)

(36b) [...] llevaba unos tenis gastados de tanto andar, unos tenis que en el círculo de los hijos de algunas de mis amistades, estarían desde hace mucho tiempo sepultados en el closet o abandonados en un basurero.

El mismo tipo de fenómenos se da, por último, en las construcciones abstractas de resumen. Véanse (37a)-(39a) y sus transformaciones posibles en (37b)-(39b):

(37a) La vigilancia debe dirigirse, no sólo a la fiscalización de los dineros de la Institución en la forma usual, sino a velar porque ésta sea administrada con criterio mercantil. En resumen, la supervisión de los órganos controladores del Estado, tendrá también que verificar si la Institución está bien manejada y produce para el Estado los beneficios adecuados.

(CORPES XXI, Rodil Rivera Rodil, La Marina Mercante de Honduras, Lithopress, Tegucigalpa, 2001) 
(37b) La supervisión de los órganos controladores del Estado, tendrá también que verificar si la Institución está bien manejada y produce para el Estado los beneficios adecuados.

(38a) Además la competencia por mercado también será importante, ya que por ejemplo Ariane Space cobra 6,600 dólares por libra de carga, mientras que nosotros 2,600 dólares, en resumen GlobalSat cobra 1.8 mdd mientras que el Ariane cobra 140 millones de dólares, a ello tenemos que añadir la experiencia en lanzamientos con la que contamos.

(CORPES XXI, «Jalisco y Quintana Roo, ¿Plataformas de Lanzamiento de Satélites?», Excélsior, México D. F.: excelsior.com.mx, 25-09-2001)

(38b) Además la competencia por mercado también será importante, ya que por ejemplo GlobalSat cobra 1.8 mdd mientras que el Ariane cobra 140 millones de dólares, a ello tenemos que añadir la experiencia en lanzamientos con la que contamos.

(39a) con la posibilidad de entrar a formar parte de ese grupo de bichitos insignificantes, de convertirse en una molécula voladora con la libertad de pasar desapercibida, de poder marcharse haciendo mutis, de volatilizarse, en resumen.

(CORPES XXI, Lola Beccaria, La luna en Jorge, Destino, Barcelona, 2001)

(39b) con la posibilidad de volatilizarse.

Como ya señalamos, no nos ocupamos en este trabajo del estudio de las construcciones concretas de reformulación desde un punto de vista sintáctico, pero sí nos parecen inevitables unas mínimas consideraciones sobre esta faceta del comportamiento sintáctico de las construcciones de reformulación, en la que deben ser analizadas como basadas en relaciones de interdependencia ${ }^{62}$, toda vez que su condición específica de unidades de reformulación exige la presencia simultánea de dos elementos, RDO y RDOR. La estructura de las unidades concretas de reformulación, en los niveles A, SA y UDE respondería, por consiguientes a un esquema como el de (40):

${ }^{62}$ Se produciría en este punto una coincidencia, aunque solo aparente, con los planteamientos metodológicos de E. Roulet en su segundo modelo, en el que las relaciones sintácticas entre las unidades discursivas son de tres tipos, que denomina de independencia, dependencia e interdependencia, y los de J. Portolés, que desarrolla y concreta esta clasificación, considerando que la reformulación se basa en una relación de interdependencia entre los constituyentes inmediatos de la construcción. Al no diferenciar ambos autores entre los planos abstracto y concreto de una construcción sintáctica a que hemos hecho referencia en nuestro trabajo, la aplicación de esta tipología relacional no escapa a contradicciones. Véanse E. Roulet «Le modèle genevois d'analyse du discours: évolution et perspectives», Pragmatics, 1, 1991, págs. 243-248, y J. Portolés, «Sobre la organización interna de las intervenciones», en A. Briz et alii, Pragmática y gramática del español hablado, Universidad de Valencia, Departamento de Filología Española, 1996, págs. 203-214. 
(40)

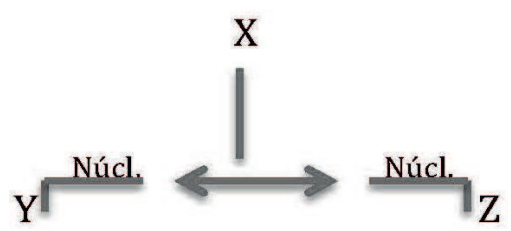

\section{Conclusiones}

Nuestro trabajo sobre la estructura sintáctica de las construcciones de reformulación permite poner de manifiesto dos series diferentes de hechos. De una parte, en un plano general, la posibilidad y utilidad de una sintaxis del discurso basada en los mismos principios generales que han mostrado su operatividad en la descripción/ explicación de la sintaxis oracional: fundamentalmente, la consideración de las unidades y las relaciones sintácticas discursivas a la luz de la distinción entre elementos abstractos / elementos concretos, y la descripción de las relaciones sintácticas como equivalentes de las funciones hjelmslevianas. De otra, en un plano específico, con tales planteamientos, la caracterización sintáctica de las construcciones de reformulación como construcciones abstractas de estructura coordinada.

Esta propuesta de análisis ofrece una visión de la reformulación que se separa ampliamente de la postulada en otros modelos. En primer lugar, se centra en el estudio de las relaciones sintácticas que se producen entre los constituyentes inmediatos de las construcciones de reformulación, no en el de los marcadores presentes en ellas. Considera, por tanto, que los mecanismos de reformulación descansan sobre estructuras sintáctico-discursivas que son independientes de los marcadores de reformulación, y que estos vendrían a sumarse, con su significado, al de los esquemas sintácticos coordinados correspondientes, que pueden/deben ser definidos independiente y específicamente por sus relaciones funcionales en los planos abstracto y concreto.

Nuestro análisis presenta una ventaja de carácter explicativo frente a otras posiciones metodológicas reseñadas: permite ofrecer una visión unitaria de las construcciones de reformulación, al remitirlas a un único tipo de estructura sintáctica abstracta; un único esquema sintáctico donde los constituyentes coordinados, RDO y RDOR presentan una autonomía funcional que estaría en correlación con lo que, en la línea de argumentación de Cristofaro ${ }^{63}$ (2003) para las construcciones subordinadas, podríamos denominar, para el caso de las coordinadas, principio de asunción de simetría; un principio con el que se resaltaría la simetría o equivalencia nuclear de los constituyentes inmediatos de estas construcciones, en cada uno de los niveles en que estas operan, y respecto de las propiedades características de cada nivel. Así, la simetría de RDO y RDOR

${ }^{63}$ Véase S. Cristofaro, Subordination, Oxford University Press, 2003. 
en el nivel de las UDE se traduciría en la denotatividad, o capacidad denotativa directa y autónoma, de cada uno de los UDE de la expresión reformulada; la simetría de los constituyentes SA de un acto, en la asertividad equivalente de cada uno de los SA reformulados, y la de los A de una I, en la ilocutividad plena equivalente de cada uno de tales actos reformulados.

El análisis de la reformulación como una operación sintácticamente basada en construcciones coordinadas nos permite entender el modo en que el emisor de una comunicación puede dar cumplimiento, y hacerlo en forma comprensible para su interlocutor, a su voluntad reformuladora: insatisfecho con una primera formulación, puede, gracias a la existencia de los mecanismos sintácticos de coordinación, completar su enunciado coordinando a dicha primera formulación otra estructuralmente equivalente a la primera, capaz, por ello, de transmitir una nueva denotación elemental de valor equivalente al de la primera, cuando se reformula una UDE; de transmitir una nueva aserción de valor equivalente a la anterior, cuando se reformula un SA, y de transmitir un acto ilocutivo de valor equivalente al anterior, cuando se trata de reformular un A. 